\title{
A Model for Two-Dimensional Compaction of Cylinders
}

\author{
Takayasu IKEGAMI \\ (National Institute for Research in Inorganic Materials, 1-1, Namiki, Tsukuba-shi, Ibaraki \\ 305)

\section{円柱の 2 次元的圧密モデル} \\ 池上隆康 \\ (無機材質研究所, 305 茨城県つくば市並木 1 -1)

\begin{abstract}
A model for two-dimensional compaction of cylinders is proposed. The equation $\ln \left(P_{2} / P_{1}\right)=k_{1} k_{s} N_{t}\left(1 / Z_{s 2}-1 / Z_{s 1}\right)$ based on the model well described an experimental relation between the applied pressure $P_{a}$ and the number of propagation sources of rearrangement, $Z_{s}$, where $k_{t}$ is a constant, $k_{s}$ is a coefficient of friction, $N_{t}$ is the total number of contacts among cylinders, $Z_{s 1}$ and $P_{1}$ are the initial value of $Z_{s}$ and that of $P_{a}$, and $Z_{s 2}$ and $P_{2}$ are the final ones, respectively. The equation indicates that $\ln \left(P_{2} / P_{1}\right)$ is roughly proportional to $1 / Z_{s 2}$ under the condition $P_{I} \ll P_{2}$ because of $Z_{s 2} \ll Z_{s l}$, which means that a feature in the initial packing structure vanishes on compaction. The $k_{s}$ value, on the other hand, has appreciable influence on the $Z_{s z}$ value, i.e., the final packing structure.

[Received December 18, 1989; Accepted April 13, 1990]
\end{abstract}

Key-words : Theory of compaction, Two-dimensional compaction, Compaction of cylinders, Compaction apparatus

\section{Introduction}

There is an essential difference between a regular array of particles in a sintering model and a random one in an actual powder compact. This causes severe questions ${ }^{1 /-3 !}$ for application of theoretical equations of sintering to practical data of a usual powder. If one can elucidate packing structure of particles in a usual powder compact, a sintering theory must be effectively developed.

Successive studies ${ }^{4)-10\}}$ about compaction have been made in various fields such as ceramics, ${ }^{41}$ powder metallurgy, ${ }^{51}$ soil ${ }^{61.7)}$ pharmacy $^{81}$ and applied physics. ${ }^{9 / 10)}$ Compaction phenomena of a powder are so complicated that most of the studies have derived only empirical equations relating an applied pressure to the volume of a powder compact. Mathematical studies, ${ }^{9,10)}$ on the other hand, have been made under an assumption that a powder compact can be treated as a continuum medium. This assumption is reasonable for macroscopic behaviors ${ }^{11)}$ of a powder. It is, however, well-known that properties of ceramics critically subject not only to macroscopic inhomogeneity ${ }^{121}$ in packing of particles but also to packing defects ${ }^{13\}}$ of particles in microscopic regions. The present study was made to obtain information about microscopic packing structure of particles. Since it is very difficult to analyze and describe a three-dimensional packing structure of particles, a two-dimensional one was examined through analysis of compaction process of clinders located in the flat box of a compaction apparatus. ${ }^{14}$

\section{A compaction model}

\subsection{Typical arrays of cylinders}

Figure 1 shows two typical examples of packing of cylinders, from which one can find some statistical relation between a number of cylinders $C_{p}$ and the shape of a polygon linked with their centers. Figure 2 shows four examples of the polygons, in which polygons with $C_{\mathrm{p}} \leq 5$ are inevitably convex. If a concave polygon is defined as the polygon having one concave site at least, a number fraction, $R_{\mathrm{c}}$, of the total number of concave polygons for a given $C_{\mathrm{p}}$ abruptly increases with increasing the $C_{p}$ value from 6 . Most of polygons of $C_{\mathrm{p}} \geq 8$ are accordingly concave. Figure 2 shows that when cylinder a or $b$ at a convex site shrinks toward the center of the polygon, cylinders $\mathrm{c}, \mathrm{d}, \cdots$ near it inevitably move toward outer side. The applied pressure restricts such movement in general. The polygons, therefore, are considered to be hard in the sense that the restriction force prevents them from breaking down. It is, on the other hand, probable that cylinder $e$ or $f$ at a concave site in Fig. 2 shrinks toward the center of the polygon together with cylinders $g, h, \cdots$ near it. The concurrent 

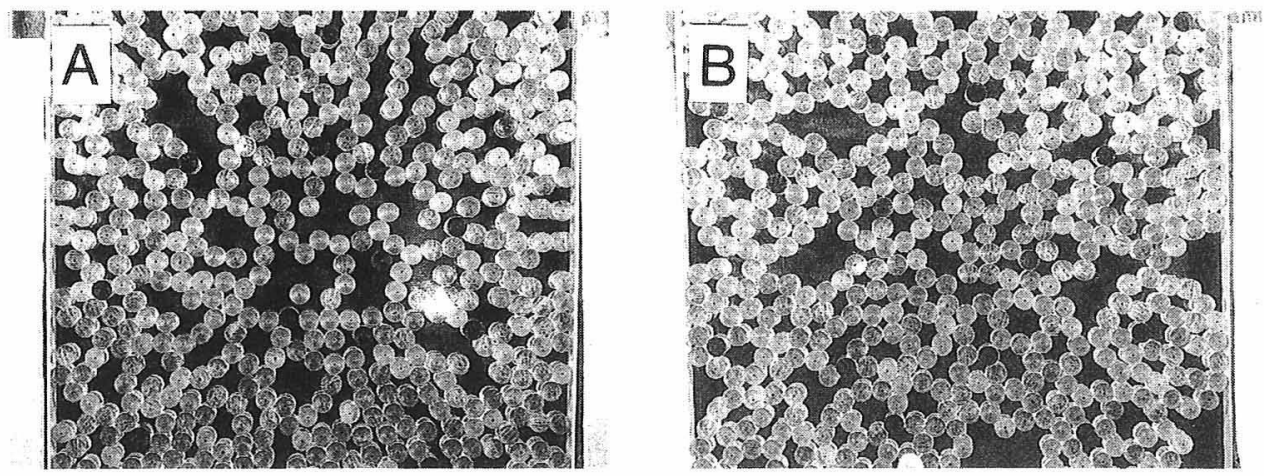

Fig. 1. Two typical arrays of adhesive cylinders. (A) random, (B) granules
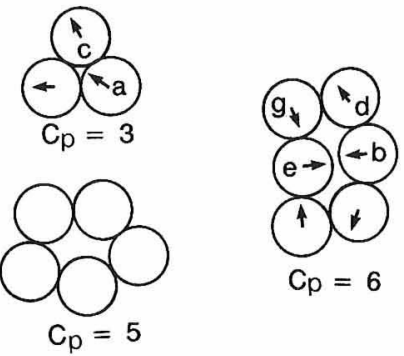

Fig. 2. Four examples of polygons linked with $C_{\mathrm{p}}$ cylinders.

shrinkages indicate no operation of the restriction force stated above, and hence the polygon is considered to be soft in the sense that its collapse can easily occur. Furthermore, most concave sites in a polygon can play the role of propagation sources, PS's, at the rearrangement of cylinders.

\subsection{Rearrangement of cylinders in a sub- region}

We consider a slide at a contact between two cylinders. Such a slide occurs when an applied force, $F_{\mathrm{a}}$, increases by $\Delta f_{\mathrm{a}}$. Physics ${ }^{6), 71.9)}$ lead an equation,

$$
\Delta f_{\mathrm{a}}=k_{\mathrm{s}} F_{\mathrm{a}}
$$

where $k_{\mathrm{s}}$ is a coefficient of friction. A sub-region is defined as a region where the rearrangement of cylinders is directly induced by that at one or several PS's. If simultaneous slides occur at $Z_{\mathrm{r}}$ contacts in the sub-region, $Z_{\mathrm{r}} \Delta f_{\mathrm{a}}$ is equal to the least increment of $F_{\mathrm{a}}$ which just causes the rearrangement. There is a relation,

$$
P_{\mathrm{a}}=n_{\mathrm{a}} F_{\mathrm{a}}
$$

among $F_{\mathrm{a}}$, an applied pressure, $P_{\mathrm{a}}$, and a number of cylinders per a unit length for the planar packing, $n_{\mathrm{a}} . n_{\mathrm{a}}$ is statistically equal to $1 /\left(\sqrt{\rho_{\mathrm{r}}} \times\right.$ $d_{\mathrm{c}}$ ), where $\rho_{\mathrm{r}}$ is an area fraction of occupation of cylinders to the total area of the compaction field and $d_{\mathrm{c}}$ is the radius of a cylinder. The change of $P_{\mathrm{a}}$ extends over several orders in general. The previous study, ${ }^{14}$ on the other hand, showed the practically slight change of the $\rho_{\mathrm{r}}$ value from $60 \%$ to $80 \%$. This inappreciable change as compared with the former one roughly means that $n_{\mathrm{a}}$ is constant during compaction. Consequently, Eq. (2) indicates that $F_{\mathrm{a}}$ is propotional to $P_{\mathrm{a}}$. It is natural that $Z_{\mathrm{r}} \Delta f_{\mathrm{a}}$ is induced by increase, $\Delta P_{\mathrm{r}}$, of $P_{\mathrm{a}}$. From these relations and Eq. (1),

$$
\Delta P_{\mathrm{r}}=k_{\mathrm{s}} Z_{\mathrm{r}} P_{\mathrm{a}}
$$

is obtained.

\subsection{Decrease of PS in number}

To derive an equation which relates a number of sub-regions to an applied pressure, the following are assumed:

(1) An applied pressure, $P_{\mathrm{a}}$, results in some balanced array of cylinders. At this time, force $F_{\text {a }}$, which is proportional to $P_{\mathrm{a}}$, acts at a contact between cylinders.

(2) The rearrangement of cylinders at PS has directly influence on directions of movement of other cylinders in the same sub-region, but has indirectly influence on those in the other subregions.

(3) A number, $Z_{\mathrm{r}}$, of sliding contacts in a sub-region is proportional to the total number of contacts $Z_{\mathrm{m}}$ in it.

Compaction induces translations or rotations of cylinders and their clusters with slides at contacts among cylinders as well as those without such slides. $Z_{\mathrm{r}}$, therefore, is smaller than $Z_{\mathrm{m}}$. It is very difficult exactly to estimate the $Z_{\mathrm{r}}$ value. If, however, rearrangement of cylinders is fully random, a constant ratio is statistically evaluated between sliding cylinders and the other ones. This situation is in accord with assumption ( 3 ).

From the definition, every sub-region has a PS or more PS's. PS's which exist closely must belong to the same sub-region, and co-operatively induce the rearrangement of cylinders in it. The existence of such sub-regions means that the total number, $Z_{\mathrm{s}}$, of sub-regions is not more than that, $Z_{\mathrm{p}}$, of PS's. Equation ( 3 ), however, indicates that the $\Delta P_{\mathrm{r}}$ value decreases with decreasing the $Z_{\mathrm{r}}$ value. This tendency means that rearrange- 
ment of cylinders easily occurs in a compaction field with small (many) sub-regions in comparison with that with larger (fewer) ones. This condition suggests that sub-regions with one PS are much more than the other with several PS's in general, and $Z_{\mathrm{s}}$ is roughly estimated as $Z_{\mathrm{p}}$. The compaction field is, thus, divided into $Z_{\mathbf{p}}$ subregions from PS to PS.

Assumption ( 1 ) indicates that the increase of $P_{\mathrm{a}}$ to $P_{\mathrm{a}}+\Delta P_{\mathrm{r}}^{\prime}$ results in a new balanced array of cylinders with PS's of $Z_{s}-1$ from the old one with $Z_{\mathrm{s}}$. Rearrangement from $\left(Z_{\mathrm{s}}-1\right)$ to $\left(Z_{\mathrm{s}}-2\right)$ occurs if the applied pressure increases from $P_{\mathrm{a}}+$ $\Delta P_{\mathrm{r}}^{\prime}$ to $P_{\mathrm{a}}+2 \Delta P_{\mathrm{r}}^{\prime}+\Delta_{1}$, further one from $\left(Z_{\mathrm{s}}-2\right)$ to $\left(Z_{\mathrm{s}}-3\right)$, also, proceeds after increase of the applied pressure from $P_{\mathrm{a}}+2 \Delta P_{\mathrm{r}}^{\prime}+\Delta_{1}$ to $P_{\mathrm{a}}+$ $3 \Delta P_{\mathrm{r}}^{\prime}+\Delta_{2}$, and so on. If $\mathrm{d} Z_{\mathrm{s}}-1 \ll Z_{\mathrm{s}}, \Delta_{q} \ll \Delta P_{\mathrm{r}}^{\prime}$ $(q=1,2, \cdots) . \mathrm{d} P_{\mathrm{a}}$ is defined as the increment of the applied pressure during which the value of $Z_{\mathrm{s}}$ decreases by $\mathrm{d} Z_{\mathrm{s}} . \mathrm{d} P_{\mathrm{a}}=\Delta P_{\mathrm{r}}^{\prime} \mathrm{d} Z_{\mathrm{s}}$. Very difficult estimation of the $\Delta P_{\mathrm{r}}^{\prime}$ value results in that of the $\mathrm{d} P_{\mathrm{a}}$ value. A relation that $\Delta P_{\mathrm{r}}^{\prime}$ is proportional to $\Delta P_{\mathrm{r}}$, however, may hold because of slight structural change of array of cylinders from $Z_{\mathrm{s}}$ to $Z_{\mathrm{s}}-1$. Both the values of $Z_{\mathrm{s}}$ and $\mathrm{d} Z_{\mathrm{s}}$ are proportional to the area of the compaction field, which, on the other hand, has inconsiderable influence on the value of $\mathrm{d} Z_{\mathrm{s}} / Z_{\mathrm{s}} . \mathrm{d} P_{\mathrm{a}}$ is hence related to $\mathrm{d} Z_{\mathrm{s}} / Z_{\mathrm{s}}$ to eliminate the effect of the area of the compaction field on the relation, and $\mathrm{d} P_{\mathrm{a}}$ is found to be proportional to the product of $\Delta P_{\mathrm{r}}$ and $\mathrm{d} Z_{\mathrm{s}} / Z_{\mathrm{s}} . N_{\mathrm{t}}$ denotes the total number of contacts in the compaction field, and $Z_{\mathrm{s}}$ is equal to $N_{\mathrm{t}} / Z_{\mathrm{m}}$. From the aforesaid relations, $\mathrm{d} P_{\mathrm{a}} \alpha \Delta P_{\mathrm{r}}$ $\times \mathrm{d} Z_{\mathrm{s}} / Z_{\mathrm{s}}, \quad Z_{\mathrm{r}} \alpha Z_{\mathrm{m}}$ and $Z_{\mathrm{s}}=N_{\mathrm{t}} / Z_{\mathrm{m}}$

$$
\mathrm{d} P_{\mathrm{a}}=-k_{1} k_{\mathrm{s}} \frac{N_{\mathrm{t}}}{Z_{\mathrm{s}}} P_{\mathrm{a}} \frac{\mathrm{d} Z_{\mathrm{s}}}{Z_{\mathrm{s}}}
$$

is derived from Eq. ( 3 ), where $k_{1}$ is a constant. If $N_{\mathrm{t}}$ is a constant, integrating Eq. (4) results in,

$$
\ln \left(P_{2} / P_{1}\right)=k_{1} k_{\mathrm{s}} N_{\mathrm{t}}\left(\frac{1}{Z_{\mathrm{s} 2}}-\frac{1}{Z_{\mathrm{s} 1}}\right)
$$

where $P_{1}$ and $Z_{\mathrm{s} 1}$ are the initial values of $P_{\mathrm{a}}$ and $Z_{\mathrm{s}}$, and $P_{2}$ and $Z_{\mathrm{s} 2}$ are the final ones, respectively.

\section{Experimental}

The apparatus, $\mathrm{CA},{ }^{14)}$ was fabricated to observe compacting process of something like 500 acrylic resin cylinders with $10 \mathrm{~mm}$ in diameter by $16 \mathrm{~mm}$ in high. The cylinders were compacted with the four movable walls which slide on the flat plate of the apparatus. The purchased cylinders are slippery, and their sides were covered with a pressure sensitive adhere double coated tape to give adhsiveness to the cylinders. Analysis was carried both on a packing process of the randomly arranged cylinders and on that of granules consisting with about 25 cylinders. Packing structure was taken with a camera located above CA. An applied pressure was measured with a strain gauge. $\rho_{\mathrm{r}}$ was estimated as the ratio of the total basal area of cylinders to the area of the square encircling them.

\section{Results and discussion}

Table 1 shows $\rho_{\mathrm{r}}, P_{\mathrm{a}}$ and distributions of numbers of polygons. From the Table, increase of $P_{\mathrm{a}}$ occurred slightly from $\rho_{\mathrm{r}}=68 \%$, and appreciably from $\rho_{\mathrm{r}}=78 \%$. It was, however, very difficult to obtain a larger $\rho_{\mathrm{r}}$ value than $81 \%$.

If there is a direct interdependence among all the slides at contacts in the compaction field (this corresponds with the compaction field having one sub-region), it is very difficult to explain the reason why densification at a relatively low $\rho_{\mathrm{r}}(<$ $68 \%$ ) easily occurs even at an insignificantly applied pressure. This perplexed situation induces the conception, that is, "sub-regions".

Table 1. Applied pressures, $P_{\mathrm{a}}$, ratios of occupation, $\rho_{\mathrm{r}}$, and numbers of polygons linked with $C_{\mathrm{p}}$ cylinders for

\begin{tabular}{|c|c|c|c|c|c|c|c|c|c|c|c|c|c|c|}
\hline \multirow{2}{*}{$\mathrm{Pa}_{\mathrm{a}}$} & & \multirow{2}{*}{\multicolumn{2}{|c|}{$\rho$ r }} & \multicolumn{9}{|c|}{$\mathrm{C}$ 。 } & \multirow[b]{2}{*}{12} & \multirow[b]{2}{*}{13} \\
\hline & & & & 3 & 4 & 5 & 6 & 7 & 8 & 9 & 10 & 11 & & \\
\hline & 9 & 0. & 674 & 81 & 38 & 14 & 24 & 11 & 12 & 6 & 7 & 3 & 0 & 1 \\
\hline 2 . & 5 & 0. & 698 & 89 & 39 & 17 & 27 & 14 & 15 & 5 & 4 & 2 & & \\
\hline 5. & 8 & 0. & 730 & 104 & 45 & 24 & 32 & 17 & 8 & 3 & 3 & 1 & & \\
\hline 7. & 7 & 0. & 739 & 112 & 62 & 26 & 32 & 18 & 5 & 2 & 2 & & & \\
\hline 14. & 2 & & 760 & 131 & 70 & 34 & 32 & 12 & 3 & 3 & 1 & & & \\
\hline 20. & 4 & 0. & 770 & 153 & 71 & 36 & 24 & 15 & 2 & 3 & & & & \\
\hline 22. & 6 & 0. & 780 & 177 & 66 & 38 & 21 & 14 & 2 & 2 & & & & \\
\hline 24. & 0 & 0. & 793 & 189 & 74 & 35 & 21 & 14 & 1 & 2 & & & & \\
\hline 37. & 2 & 0. & 796 & 189 & 78 & 40 & 23 & 11 & 1 & 1 & & & & \\
\hline 55. & 0 & 0. & 807 & 196 & 64 & 49 & 23 & 10 & 1 & 1 & & & & \\
\hline 87. & 0 & 0. & 807 & 206 & 71 & 47 & 20 & 9 & 1 & 1 & & & & \\
\hline 122. & 0 & 0. & 808 & 215 & 82 & 42 & 19 & 7 & 1 & 1 & & & & \\
\hline 135. & 0 & 0. & 809 & 204 & 84 & 46 & 17 & 7 & 1 & 1 & & & & \\
\hline 156. & 0 & 0. & 810 & 209 & 84 & 46 & 17 & 6 & 1 & 1 & & & & \\
\hline
\end{tabular}
a random array. 


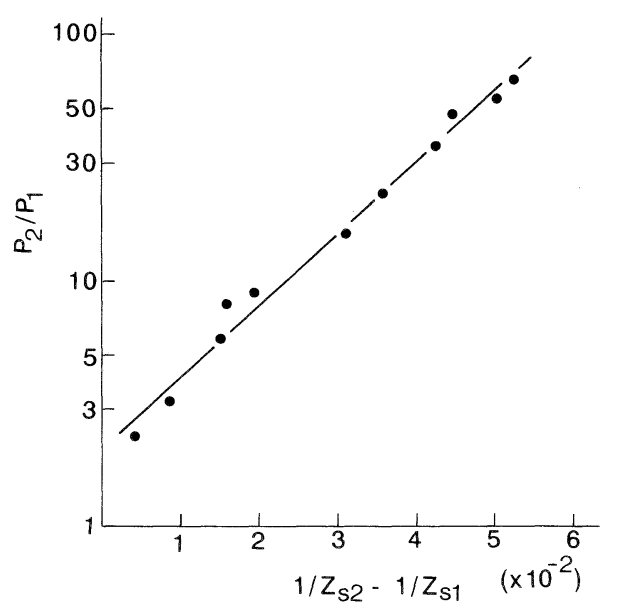

Fig. 3. A relation between $P_{2} / P_{1}$ and $1 / Z_{\mathrm{s} 2}-1 / Z_{\mathrm{s} 1}$.

This conception, also, well explains one of origins for the abrupt increase of $P_{\mathrm{a}}$ from $78 \%<\rho_{\mathrm{r}}$ because of appreciable decrease of PS in number.

Numbers of polygons of $C_{\mathrm{p}}=3$ to 5 notably increased, but those of $C_{\mathrm{p}} \geq 8$ decreased on compaction. These different tendencies in change of the number may be attributed to the shapes of polygons. That is, the polygons of $C_{\mathrm{p}}=3$ to 5 are inevitably convex, but $R_{\mathrm{c}}$ for a given $C_{\mathrm{p}}$ value increased with increasing $C_{\mathrm{p}}$, and most of polygons of $C_{\mathrm{p}} \geq 8$ are concave.

The value of $Z_{\mathrm{s}}$ has to be evaluated to test the reasonability of Eq. ( 5 ), but not only this evaluation but also that of $Z_{\mathrm{p}}$ is very difficult. The major part of concave site, on the other hand, was identified as propagation sources of rearrangement of cylinders, and $Z_{\mathrm{s}}$ may be substituted with a number of all the concave sites in the compaction field. We, then, enumerated such sites to calculate Eq. ( 5 ). The solid line in Fig. 3 shows the result obtained from Eq. ( 5 ) under an assumption that the $k_{1}$ value is constant. The close fit of the theoretical values to the experimental data supports the reasonability of this assumption.

Equation ( 5 ) indicates that the value of $1 / Z_{\mathrm{s} 2}$ $-1 / Z_{\mathrm{s} 1}$ approaches to that of $1 / Z_{\mathrm{s} 2}$ with increasing $P_{2}$. This means that if an applied pressure is appreciably high, $Z_{\mathrm{si}}$, an initial array of cylinders, has slight influence on the $Z_{\mathrm{s} 2}$ value, the final array of cylinders. Our previous paper ${ }^{14}$ has shown that the initial array of cylinders has strong influence on packing structure in the early stage of compaction. This feature vanished on compaction as suggested from Eq. ( 5 ). The data, also, roughly support that the $k_{1}$ value is constant. It was, however, very difficult completely to eliminate the trace of the packing feature in the initial array in spite of applying a very high pressure. Further development of the present compaction model is required to specify the trace.

It is well-known that a coefficient of friction ${ }^{11}$ has appreciable influence on packing structure. Equation ( 5 ), also, theoretically confirms this influence irrespective of a degree of compaction. This result suggests that it is very desirable to fabricate a powder with a very small $k_{\mathrm{s}}$ for achievement of a very small $Z_{\mathrm{s} 2}$ value, effective elimination of relatively large pores.

\section{Conclusions}

The presently derived equation describes a two-dimensional compacting process, where the initial feature in a packing array vanishes on compaction. The equation, also, indicates that a final structure by compaction statistically depends both on a friction coefficient among particles and a final compression pressure.

\section{References}

1) D. L. Johnson and T. M. Clarke, Acta Metall., 12, 1173-79 (1964).

2) K. Asaga and K. Hamano, Yogyo-Kyokai-Shi, 83, 136-42 (1975).

3) C. Greskovich and K. W. Lay, J. Am. Ceram. Soc., 55, 142-46 (1972).

4) A. R. Cooper, Jr. and L.E. Eaton, J. Am. Ceram. Soc., 45, 97-101 (1962).

5) G. C. Kuczynski and I. Zaplatynski, J. Metals, 8, 215 (1956); Trans. AIME, 206, 215 (1956).

6) R. T. Shield, J. Mechanics Phys. Solids, 4, 10-16 (1955).

7) K. Kawakita and Y. Tsutsumi, Bull. Chem. Soc. Japan, 39, 1364-68 (1966).

8) M. Nakagaki and K. Sunada, Japan J. Pharmacy, 83, 73-78 (1963).

9) T. Wakabayashi, Oyo Butsuri, 31, 556-71 (1962).

10) S. Hayakawa, Report Sci. Technol. of Tokyo Univ., 4, 87-92 (1950).

11) T. Nagao, Seramikkusu, 18, 804-09 (1983).

12) T. Ikegami and Y. Moriyoshi, J. Am. Ceram. Soc., 67, 174-78 (1984).

13) T. Ikegami, M. Tsutsumi, S. Matsuda, S. Shirasaki and H. Suzuki, J. Appl. Phys., 49, 4238-41 (1978).

14) T. Ikegami, S. Matsuda, S. Shirasaki and K. Eguchi, "Sintering 87", Proc. Int'l Institute for the Science of Sintering (IISS) Symposium, Ed. by S. Sōmiya, M. Shimada, M. Yoshimura and R. Watanabe, Elsevier Appl. Sci., Tokyo (1987) pp. 285-90. 